\section{Padrões espaço-temporais da mortalidade por câncer de pulmão no Sul do Brasil}

\section{Space-time analysis of lung cancer mortality in Southern Brazil}

Mônica Silva Monteiro de Castro

Laboratório de Estatística Espacial (LESTE)

Departamento de Estatística

Instituto de Ciências Exatas

Universidade Federal de Minas Gerais

Caixa Postal 702 - CEP 31270-901

Belo Horizonte - Minas Gerais - Brasil

mmcastro@uai.com.br

\section{Viviane Alves Vieira}

Departamento de Estatística

Instituto de Ciências Exatas

Universidade Federal de Minas Gerais

\section{Renato Martins Assunção}

Departamento de Estatística

Instituto de Ciências Exatas

Universidade Federal de Minas Gerais

\section{Resumo}

As neoplasias representam a segunda causa mais comum de mortalidade no Brasil, juntamente com as chamadas causas externas. Dentre as neoplasias, o câncer de pulmão é um dos mais freqüentes, tanto em homens quanto em mulheres, e é também um dos que apresentam maior letalidade. Além disso, o risco atribuível do tabagismo como agente etiológico deste câncer é bastante alto, o que o torna potencialmente susceptível a medidas preventivas de saúde pública. $\mathrm{O}$ objetivo deste trabalho foi analisar os padrões espaço-temporais de câncer de pulmão em quatro Estados brasileiros (Rio Grande do Sul, Santa Catarina, Paraná e São Paulo), no período de 1996 a 2000. Os valores observados foram obtidos do Sistema de Informações de Mortalidade do Ministério da Saúde. Os valores esperados foram calculados utilizando-se a técnica de padronização indireta segundo sexo e faixa etária. As unidades geográficas utilizadas foram microrregiões definidas pelo IBGE. Foi utilizado um modelo bayesiano que permite interação espaço-temporal, ajustado através do software WinBUGS. Os resultados encontrados mostraram que no sul do Brasil existe um padrão em "U” nas razões de mortalidade por câncer de pulmão para homens, além de indicar áreas específicas que apresentaram riscos mais elevados e/ou maior ritmo de crescimento. A principal hipótese para este resultado seria diferentes incidências de tabagismo, mas a inexistência desta informação de abrangência regional impediu que esta variável fosse incluída na análise. Os resultados deste artigo podem ser utilizados para instruir políticas públicas voltadas para a redução do tabagismo e da mortalidade por câncer de pulmão.

Palavras-chave: Câncer de pulmão. Mortalidade. Epidemiologia. Análise especial. Análise temporal. Métodos bayesianos. 
Abstract

Neoplasms are the second most common mortality cause in Brazil, together with injuries and accidents. Amongst the neoplasms, lung cancer is one of most frequent in men and in women, and it is also one of the most lethal. Moreover, the attributed risk of tobacco as an etiologic agent of this type of cancer is considerably high, making it potentially susceptible to preventive public health measures. The objective of this work was to analyze spatial and temporal patterns of lung cancer in four Brazilian States (Rio Grande do Sul, Santa Catarina, Paraná and São Paulo), from 1996 to 2000. The observed values were obtained from the Brazilian Ministry of Health Mortality Information System (SIM-MS). The expected values were calculated using indirect standardization according to sex and age groups (SMR). The geographic units analyzed were micro-regions defined by the Brazilian Census $\mathrm{Bu}$ reau (IBGE). A Bayesian model that allowed for space-time interaction was used, using WinBUGS software. Adjusted SMR showed that in Southern Brazil there was a "U" pattern in lung cancer mortality for men, and indicated specific areas that had higher SMR and/or greater SMR growth. The main hypothesis for this result would be different tobacco use incidences, but the absence of this information at a regional level in Brazil did not permit including this variable in our analysis. The results of this paper can be used to guide public policies directed towards reducing the use of tobacco and of mortality from lung cancer.

Key Words: Lung cancer. Mortality. Epidemiology. Spatial analysis. Temporal analysis. Bayesian methods.

\section{Introdução}

As neoplasias representavam a segunda causa de mortalidade no Brasil em 1999, juntamente com as chamadas causas externas ${ }^{1}$. Dentre as neoplasias, o câncer de pulmão é um dos mais freqüentes, tanto em homens quanto em mulheres, e é também um dos que apresentam maior letalidade. Em 1999, cerca de $12 \%$ dos óbitos por câncer no Brasil foi devido a câncer de traquéia, brônquios e pulmões, sendo esta a primeira causa de morte por câncer no sexo masculino e a segunda no sexo feminino ${ }^{2}$.

No Brasil e nos Estados Unidos, o tabagismo é a principal causa dos cânceres de pulmão, laringe, cavidade oral, faringe e esôfago ${ }^{3,4}$, sendo que nos Estados Unidos o tabagismo é responsável por cerca de um terço do total de mortes por câncer ${ }^{3}$. Além disso, o risco atribuível do tabagismo como agente etiológico do câncer de pulmão é superior a $90 \%^{2}$, o que o torna potencialmente susceptível a medidas preventivas de saúde pública, medidas essas que podem ser mais eficazes se forem direcionadas a populações específicas com base em resultados empíricos. Outros fatores etiológicos, como a exposição a poeiras minerais, sílica, asbesto e pesticidas, também foram relacionados ao câncer de pulmão no Brasil ${ }^{5}$.

A mortalidade por câncer na América Latina apresenta um padrão em que coexistem fatores de risco relacionados à pobreza e ao desenvolvimento ${ }^{6}$. O Brasil apresenta diferenças regionais marcantes, possuindo grandes áreas pouco desenvolvidas, outras desenvolvidas e outras onde coexistem as duas condições. Estas regiões apresentam taxas muito diferentes de mortalidade por câncer ${ }^{6,7}$, apresentando um padrão de crescimento aproximadamente do Norte para o Sul. Uma possível explicação para este fato é que o Rio Grande do Sul, localizado no extremo sul da região sul, é considerado um dos poucos Estados brasileiros que possuem registros considerados confiáveis de mortalidade por câncer ${ }^{5}$. Uma conseqüência importante desse gradiente norte-sul é que os números médios nacio- 
nais traduzem mal a realidade diversificada do país.

Entre 1980 e 1995, as regiões sul e sudeste do Brasil apresentaram as mais altas taxas de mortalidade por todos os tipos de câncer, excetuando-se o câncer de colo de útero. Porém, nestas duas regiões as taxas de mortalidade por todos os tipos câncer apresentaram tendência decrescente, enquanto que nas outras regiões as taxas apresentaram tendência crescente ${ }^{7}$.

Com relação ao câncer de pulmão, entre 1980 e 1995, as regiões Sul e Sudeste do Brasil apresentaram as mais altas taxas de mortalidade. Neste período, considerando o Brasil como um todo, as taxas de mortalidade por câncer de pulmão aumentaram 10,5\% entre os homens e $26,7 \%$ entre as mulheres. Estas taxas apresentaram tendência crescente em todas as regiões brasileiras, tanto em homens quanto em mulheres, com a única exceção da população masculina do Sudeste ${ }^{7}$.

A taxa de mortalidade por câncer em um dado grupo populacional depende das taxas de incidência, da sobrevida após o diagnóstico, dos riscos competitivos de morte e da composição etária da população. Os estudos de mortalidade são úteis não só para avaliar o problema do câncer em uma dada região, mas também para analisar a efetividade das estratégias de prevenção primária e secundária e a qualidade e impacto do tratamento na sobrevida dos doentes ${ }^{7}$.

A análise de padrões espaço-temporais de mortalidade ou de incidência de câncer através de técnicas bayesianas é uma ferramenta de uso recente na literatura ${ }^{8,9} \mathrm{e}$ é bastante útil para a detecção de padrões espaciais e temporais de incidência e de mortalidade de câncer e de outras doenças. Em geral, os resultados das análises bayesianas são apresentados através dos chamados mapas suavizados, que são mapas construídos a partir de taxas ajustadas, obtidas combinando-se o conhecimento da taxa de cada área com as taxas nas áreas vizinhas. Uma vantagem importante do uso de técnicas bayesianas para obtenção de mapas suavizados é que elas reduzem substancialmente a chance de obtenção de estimativas espúrias de risco alto ou baixo que indicariam falsos aglomerados de doenças. Por outro lado, estas técnicas podem levar a uma suavização excessiva dos riscos e, como conseqüência, à não detecção de áreas de risco elevado, notadamente quando esta elevação é pequena em relação à média da região $0^{9,10}$. Para o estudo do padrão espacial de risco em populações humanas não existem no momento alternativas metodológicas melhores do que os métodos bayesianos ${ }^{11,12}$, e são estes métodos que serão utilizados neste artigo.

O objetivo deste trabalho foi analisar a variação geográfica e as tendências temporais da mortalidade por câncer de pulmão em microrregiões de quatro Estados localizados na região sul e sudeste do Brasil (Rio Grande do Sul, Santa Catarina, Paraná e São Paulo), no período de 1996 a 2000.

\section{Metodologia}

\section{Dados}

Os valores para a mortalidade foram obtidos do Sistema de Informação de Mortalidade (SIM) do Ministério da Saúde e são relativos a óbitos por Neoplasia maligna da traquéia, brônquios e pulmões (referidos neste artigo de forma resumida como câncer de pulmão), segundo local de residência do indivíduo, no período de 1996 a 2000.

Devido à grande extensão geográfica do Brasil e à grande diversidade dos sistemas de atenção à saúde e de informação sobre saúde entre as regiões brasileiras, optou-se por estudar uma região geográfica menos abrangente que o país todo e que tivesse sistemas de saúde com registros de melhor qualidade. Assim, a região escolhida foi o Estado de São Paulo, que pertence à região sudeste, e a região sul, que engloba os Estados do Rio Grande do Sul, Santa Catarina e Paraná.

Como o câncer é um evento raro, para evitar instabilidades nas taxas em pequenos municípios ${ }^{11}$ optamos por trabalhar com microrregiões, que são agrupamentos de municípios definidos pelo IBGE. Os valores esperados foram calculados utilizando-se a técni- 
ca de padronização indireta segundo sexo e as seguintes faixas etárias: menor ou igual a 49 anos, de 50 a 59 anos, de 60 a 69 anos, 70 a 79 anos e 80 anos ou mais, utilizando-se como referência as taxas específicas por sexo e faixa etária da população total dos Estados.

Foi utilizado um modelo bayesiano que permite interação espaço-temporal, utilizado por Bernadinelli et al. e Assunção et al. ${ }^{13,14}$, como descrito na próxima seção. O software utilizado foi o WinBUGS ${ }^{15}$.

\section{Análise Estatística}

Um procedimento clássico para lidar espacialmente com taxas de mortalidade de doenças consiste em mapear as Razões de Mortalidade Padronizadas (Standardized Mortality Ratio - SMR, em inglês). Essas razões são as estimativas de máxima verossimilhança do risco relativo de mortalidade da doença nas áreas, considerando que a contagem dos óbitos segue o modelo de Poisson. Mais formalmente, supõe-se que

$$
\left[\mathrm{X}_{\mathrm{i}} \mid \mathrm{E}_{\mathrm{i}}, \xi_{\mathrm{i}}\right] \sim \operatorname{Poisson}\left(\mathrm{E}_{\mathrm{i}} \times \xi_{\mathrm{i}}\right)
$$

onde $\mathrm{X}_{\mathrm{i}}$ é a contagem dos óbitos da doença na área $i, \mathrm{E}_{\mathrm{i}}$ é o número esperado de óbitos na área $i$, $\xi_{\mathrm{i}}$ é o risco relativo de óbito da doença na área $i, \mathrm{i}=1, \ldots, \mathrm{N}$.

Supondo independência entre as contagens, o estimador usual de $\xi i$ é a SMR, dada por

$$
\hat{\xi}_{i}=S M R_{i}=\frac{X_{i}}{E_{i}}
$$

Entretanto, quando as áreas de investigação são pequenas e a doença estudada érara, os mapas usando SMR podem ser bastante afetados pela variabilidade nessas estimativas, sendo dominados pelas taxas de áreas pouco populosas. De fato, em áreas com baixa população, qualquer mudança mínima no número de casos da doença acarretará uma mudança considerável no SMR. No entanto, essa mudança no número de casos pode ser devido à mera flutuação aleatória ${ }^{11}$.

A alternativa para lidar com esse problema foi primeiramente proposta por Clayton
$\&$ Kaldor ${ }^{16}$ para modelar o risco relativo através do procedimento bayesiano. A idéia era impor uma estrutura de relação espacial plausível entre as áreas, modelando os riscos relativos conjuntamente como um processo espacial. Em outras palavras, usar a informação das áreas vizinhas a uma certa área para estimar seu risco relativo. Esse procedimento evita a instabilidade das estimativas baseadas somente no SMR daquela área, já que usa a informação sobre o que acontece em sua vizinhança. $\mathrm{O}$ critério utilizado para a definição de vizinhança foi a de adjacência: áreas que fazem fronteira umas com as outras foram consideradas vizinhas.

Introduzindo o componente tempo na análise, chamaremos de $\mathrm{X}$ a matriz de dimensão 157 por 5 cujos elementos são a contagem dos óbitos, cada linha representando uma área e cada coluna, um ano. Assim, o elemento $\mathrm{X}_{\mathrm{it}}$ da matriz $\mathrm{X}$ representa o número de óbitos na área i no tempo $t$. A matriz $\mathrm{E}$, definida da mesma maneira, contém a informação sobre o número de óbitos esperados das 157 microrregiões nos cinco anos. A matriz $\xi$ é a matriz dos riscos relativos de ocorrência de óbitos por câncer de pulmão, sendo definida de forma similar à Xe E. Desse modo, os elementos $\mathrm{E}_{\mathrm{it}} \mathrm{e} \xi_{\mathrm{it}}$ representam, respectivamente, o número esperado de óbitos e o risco relativo na área $i$ no tempo $t$. Aqui, $i$ varia de 1 até 157 e $t$, de -2 a 2, correspondendo aos anos 1996 a 2000.

Assim, o novo modelo é

$\left[\mathrm{X}_{\mathrm{it}} \mid \mathrm{E}_{\mathrm{it}}, \xi_{\mathrm{it}}\right] \sim$ Poisson $\left(\mathrm{E}_{\mathrm{it}} \times \xi_{\mathrm{it}}\right)$

Para acompanhar a evolução das taxas de uma mesma área ao longo dos anos e fazer projeções para os anos subseqüentes, propusemos modelar o logaritmo dos riscos relativos, $\theta_{\mathrm{it}}=\ln \left(\xi_{\mathrm{it}}\right)$, ao invés dos riscos diretamente. Essa transformação do parâmetro de interesse é muito comum em estudos estatísticos, pois, se trabalhássemos $\operatorname{com} \xi_{\text {it }}$ diretamente, seria preciso definir modelos que impedissem esse parâmetro de assumir valores impossíveis, tais como negativos ou zero. Ao trabalhar com seu logaritmo, podemos usar modelos irrestritos 
nos valores atribuídos às co-variáveis e seus parâmetros.

O modelo adotado foi

$\theta_{\mathrm{it}}=\ln \left(\xi_{\mathrm{it}}\right)=\alpha_{\mathrm{i}}+\beta_{\mathrm{i}} t, i=1, \ldots, 157$ e $t=-2,-1,0$, 1,2 ,

O parâmetro $\alpha_{i}$ representa o logaritmo do risco relativo da área $i$ no ano $t=0$, ou seja, 1998. Microrregiões com $\alpha$ menores que 1 possuíam em 1998 um risco menor que $o$ valor médio de toda região estudada e as microrregiões com $\alpha$ maiores que 1 possuíam em 1998 um risco mais elevado que o risco médio de toda região.

O parâmetro $\beta_{\mathrm{i}}$ representa o ritmo de crescimento ou decrescimento anual do logaritmo do risco relativo na área $i$. Microrregiões com valores negativos de $\beta$ tiveram decrescimento anual do risco de óbito por câncer de pulmão; em contrapartida, as microrregiões que possuem $\beta$ positivo apresentaram crescimento do risco anual.

Para os parâmetros $\alpha$ e $\beta$ foi escolhida uma distribuição a priori espacial que permite que áreas próximas no espaço tenham valores semelhantes ${ }^{14}$. Detalhes sobre o processo de estimação dos modelos e uma discussão sobre as opções acerca da forma de dependência espaço-temporal, podem ser encontrados em Bernadinelli et al. e Assunção et al..$^{13,14}$. O modelo implementado no WinBUGS ${ }^{15}$, que pode ser visto no apêndice, permitiu ainda investigar o percentual de crescimento das taxas em cinco anos, e a probabilidade do SMR superar 1,5 para cada uma das 157 microrregiões envolvidas no estudo. Para fins de mapeamento, todos os resultados encontrados foram multiplicados por 100 .

\section{Resultados}

As razões de mortalidade padronizada (SMR) de cada micro-região para cada ano estudado podem ser vistas na Figura 1 para homens e na Figura 2 para mulheres. No caso dos homens, as maiores razões foram encontradas no Rio Grande do Sul, com destaque para o sul do Estado nos dois primeiros anos e para o sudeste do Estado nos anos subseqüentes. Para mulheres, as maiores razões foram encontradas em algumas microrregiões dispersas pela área estudada, sem um padrão espacial tão bem definido quanto entre os homens.

Os valores esperados a posteriori dos SMR ajustados pelo modelo bayesiano podem ser vistos nas Figuras 3 e 4, respectivamente, para homens e mulheres. Para homens, podemos ver de forma ainda mais evidente do que na Figura 1 a concentração de taxas no sul do Rio Grande do Sul, com destaque para o sudeste do Estado. Já em relação às mulheres, o método bayesiano permitiu a identificação de sub-regiões com SMR mais altos, tendo ocorrido uma concentração dos maiores SMR no sul do Rio Grande do Sul, desta vez com destaque para o sudoeste do Estado, e em uma região localizada no Estado do Paraná.

O efeito espacial pode ser visto nos mapas para 1998 das Figuras 3 e 4. Para homens, existiu um padrão espacial com valores mais altos nos dois extremos geográficos, norte e sul, sendo que o sul apresenta os maiores valores do mapa. No caso das mulheres, o sul da região estudada apresentou os maiores valores, seguido por uma concentração de valores elevados no centro da região.

A Figura 5 apresenta as probabilidades do SMR ajustado ser maior do que 1,5, ou seja, da micro-região ter a SMR de câncer de pulmão $50 \%$ maior do que a média dos quatro Estados estudados: pode-se ver que, para homens, doze micro-regiões (Blumenau e Tijucos em Santa Catarina e Porto Alegre, Três Passos, Lajeado-Estrela, Santa Cruz do Sul, Cachoeira do Sul, Serras do Sudeste, Campanha Meridional, Pelotas, Jaguarão e Litoral Lagunar no Rio Grande do Sul) apresentaram esta probabilidade maior do que 0,9 . Para mulheres, apenas duas micro-regiões (Campanha Ocidental e Campanha Meridional no Rio Grande do Sul) apresentam probabilidade maior que 0,9 .

O percentual de crescimento das SMR no período de cinco anos pode ser visto na Figura 6. As micro-regiões que apresentaram crescimento maior do que $50 \%$ nos cinco anos estudados foram: Concórdia - SC, 
Câncer de traquéia, bronquios

e pulmão para homens
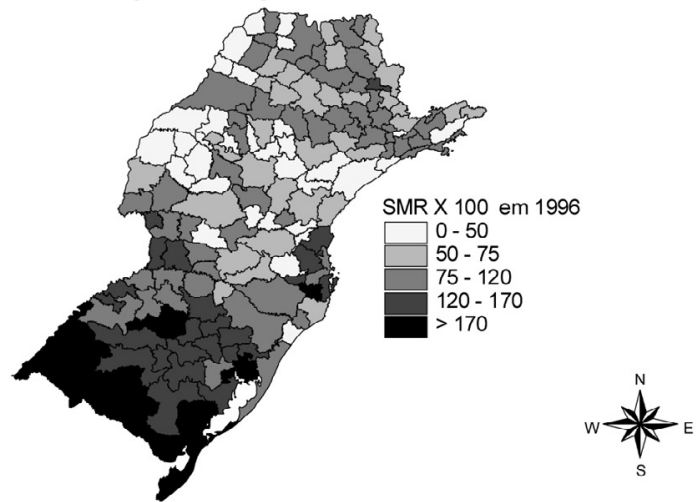

Câncer de traquéia, bronquios e pulmão para homens

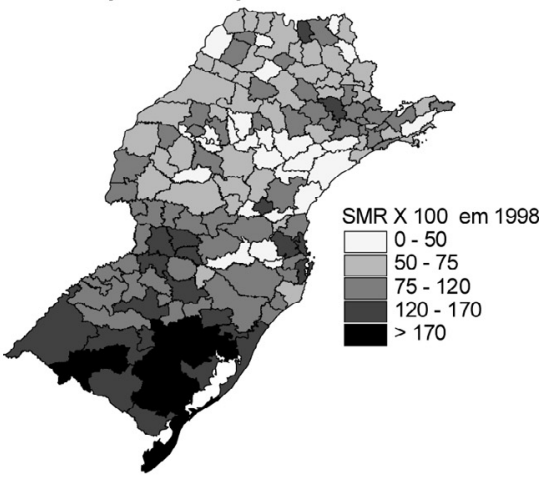

Câncer de traquéia, bronquios

e pulmão para homens

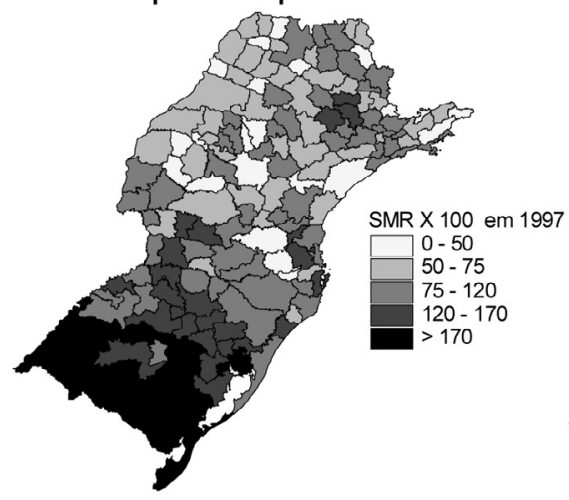

$w \frac{4_{s}^{N}}{s}$

Câncer de traquéia, bronquios e pulmão para homens

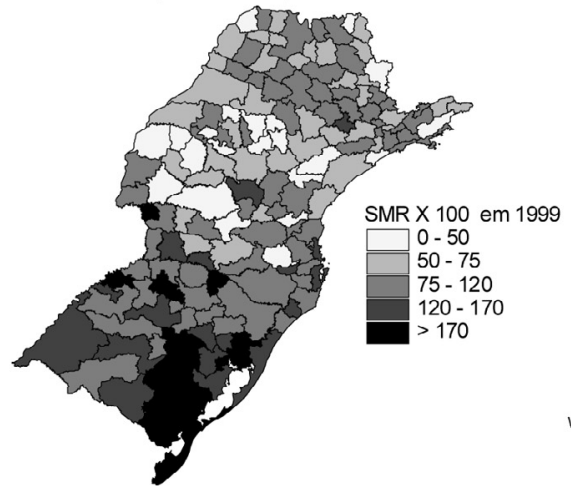

${ }_{s}^{N}$

Câncer de traquéia, bronquios

e pulmão para homens

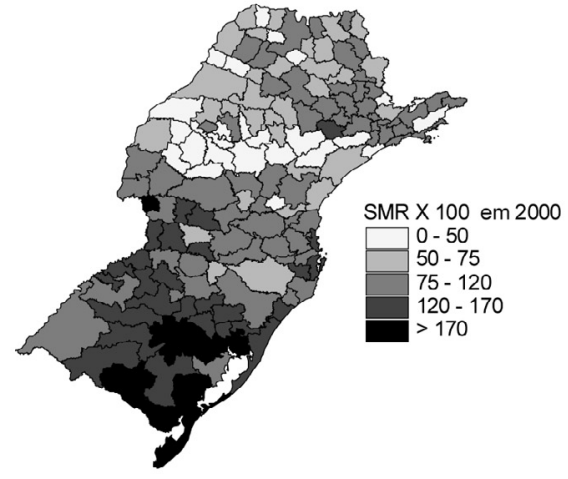

$w_{s}^{N}$

Figura 1 - Razões de Mortalidade Padronizada (SMR) brutas para homens, câncer de pulmão, São Paulo e sul do Brasil, 1996 a 2000.

Figure 1 - Crude Standardized Mortality Ratio (SMR) for men, lung cancer, São Paulo and Southern Brazil, 1996 to 2000. 
Câncer de traquéia, bronquios e pulmão para mulheres
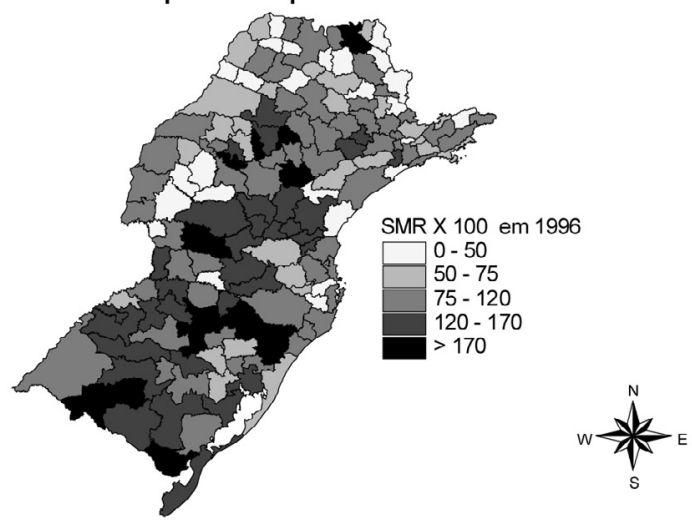

Câncer de traquéia, bronquios

e pulmão para mulheres
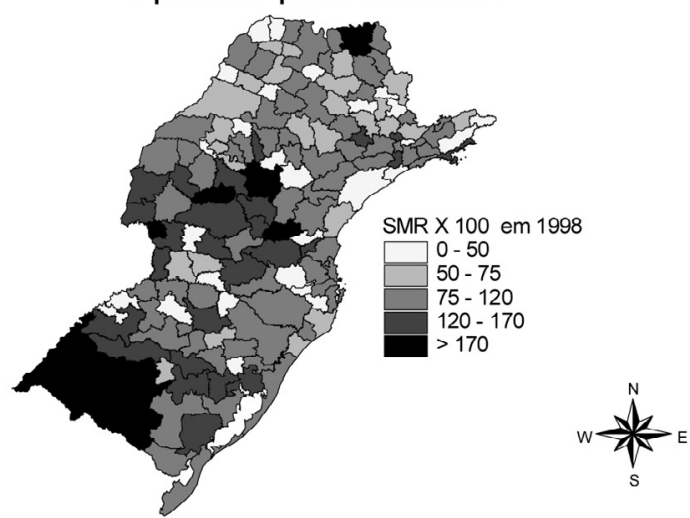

Câncer de traquéia, bronquios

e pulmão para mulheres

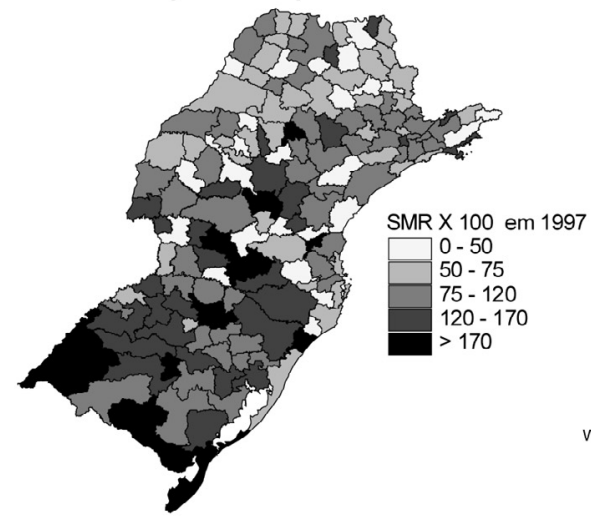

$\left.w\right|_{s} ^{N}$

Câncer de traquéia, bronquios e pulmão para mulheres

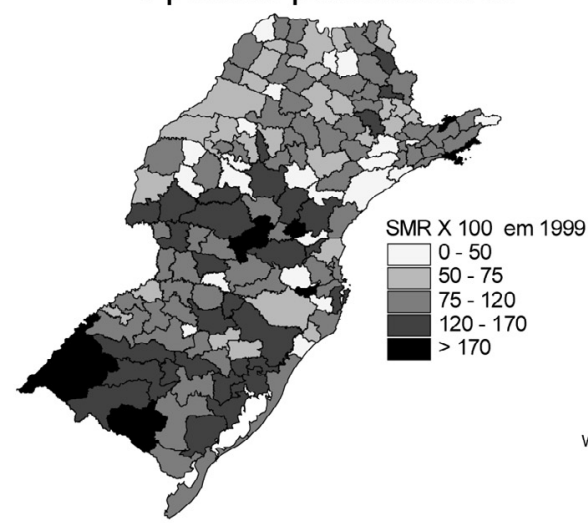

Câncer de traquéia, bronquios e pulmão para mulheres

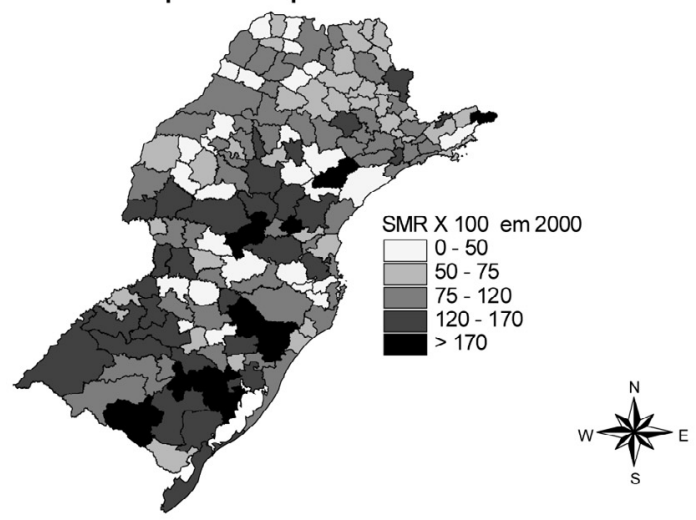

Figura 2 - Razões de Mortalidade Padronizada (SMR) brutas para mulheres, câncer de pulmão, São Paulo e sul do Brasil, 1996 a 2000.

Figure 2 - Crude Standardized Mortality Ratio (SMR) for women, lung cancer, São Paulo and Southern Brazil, 1996 to 2000. 
Câncer de traquéia, bronquios e pulmão para homens

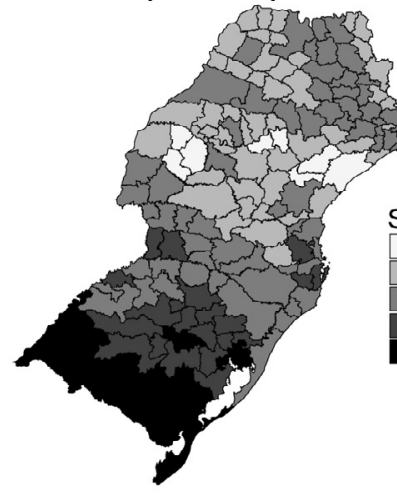

SMRAjustado X 100 em 1996

$0-50$

$50-75$

$75-120$

$120-170$

$>170$
Câncer de traquéia, bronquios e pulmão para homens

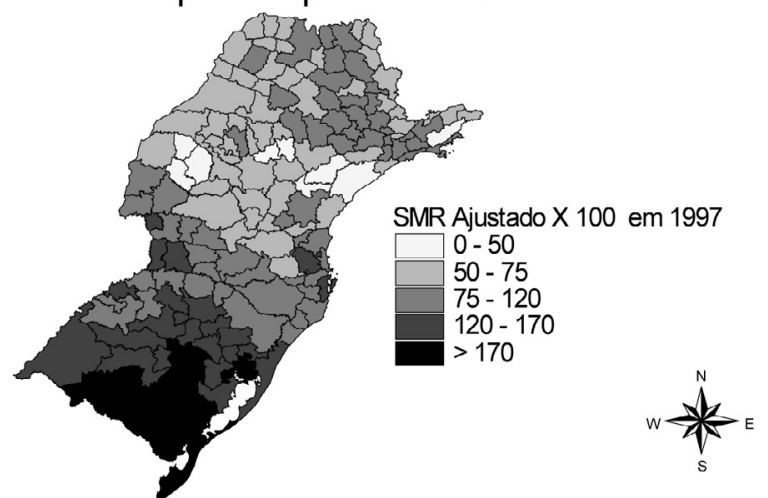

Câncer de traquéia, bronquios e pulmão para homens

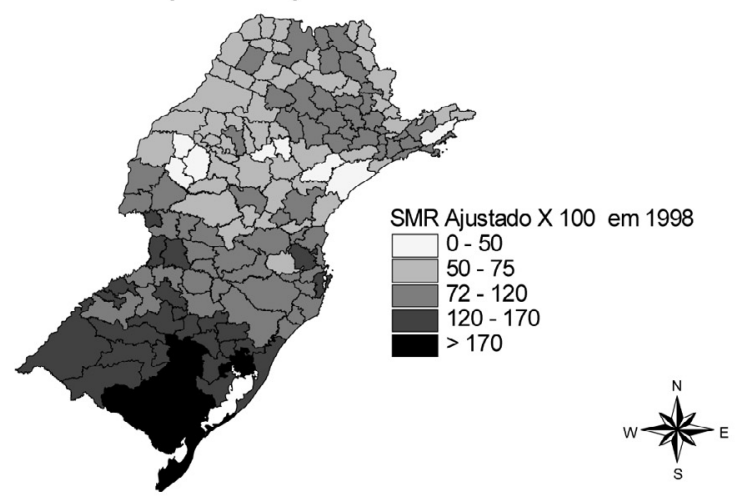

Câncer de traquéia, bronquios e pulmão para homens

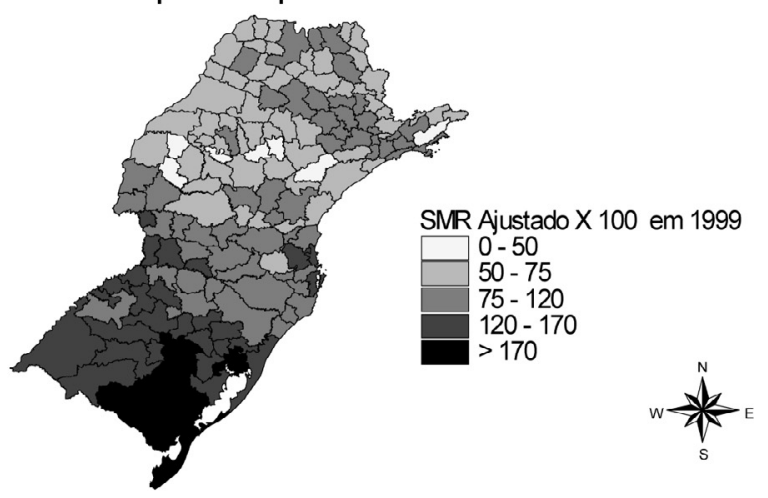

Câncer de traquéia, bronquios e pulmão para homens

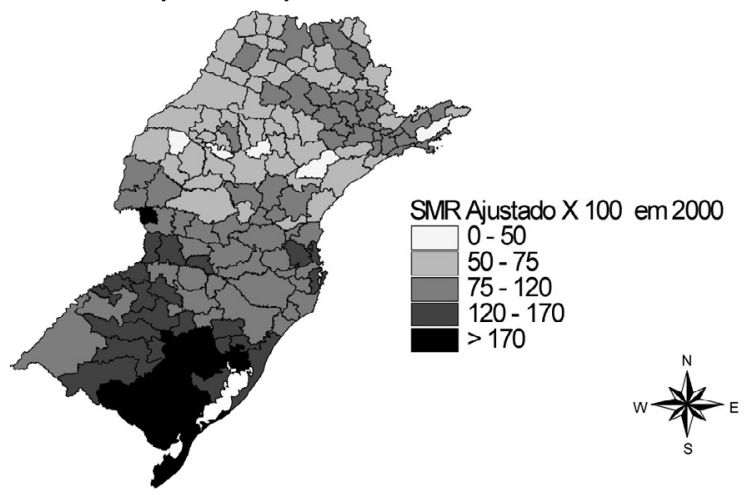

Figura 3 - Razões de Mortalidade Padronizada (SMR) ajustadas pelo modelo, homens, câncer de pulmão, São Paulo e sul do Brasil, 1996 a 2000.

Figure 3 - Adjusted Standardized Mortality Ratio (SMR) for men, lung cancer, São Paulo and Southern Brazil, 1996 to 2000. 
Câncer de traquéia, bronquios

e pulmão para mulheres

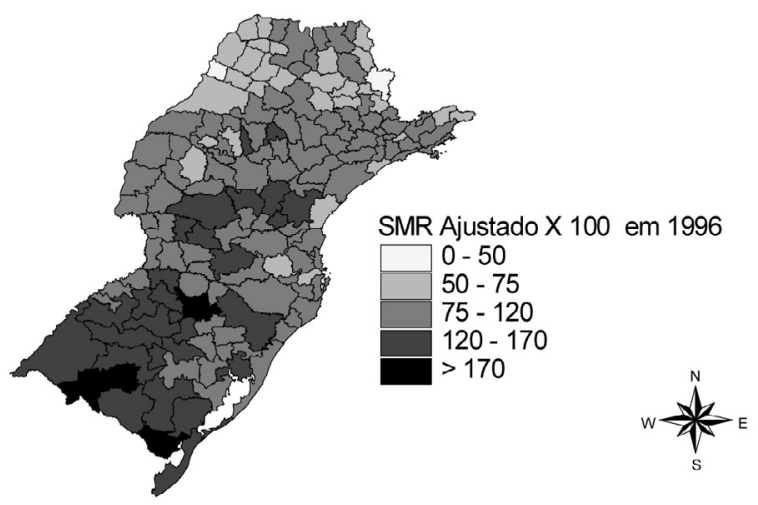

Câncer de traquéia, bronquios

e pulmão para mulheres

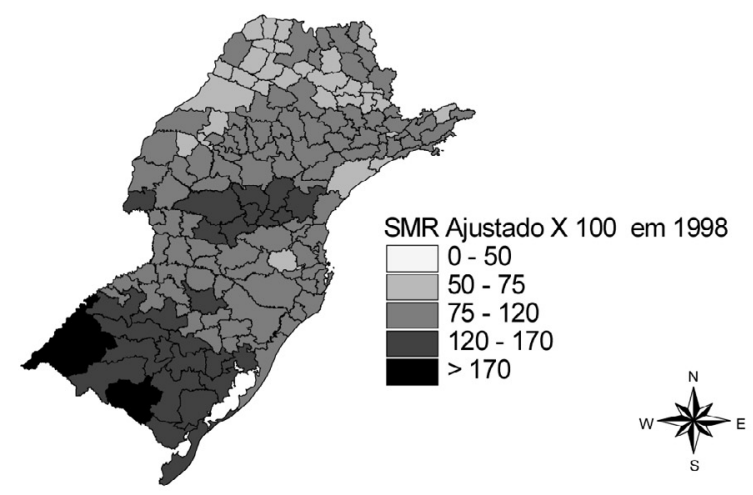

Câncer de traquéia, bronquios

e pulmão para mulheres

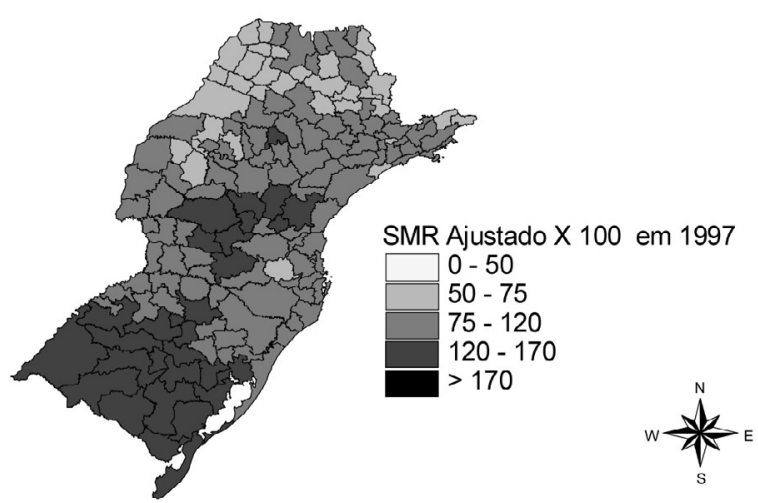

Câncer de traquéia, bronquios e pulmão para mulheres

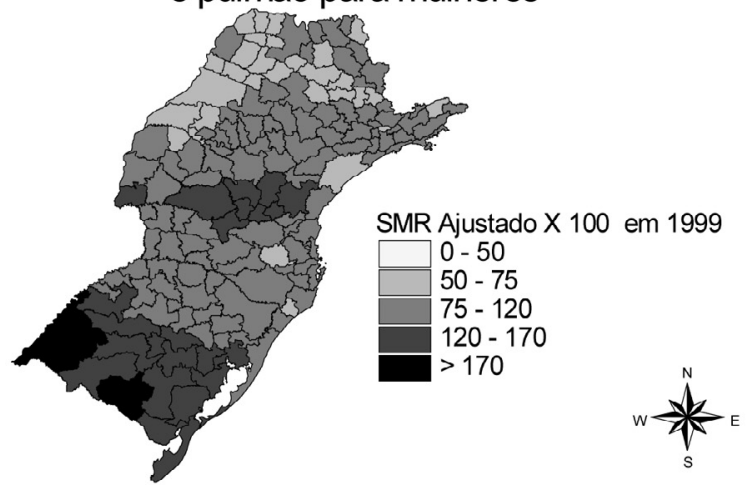

Câncer de traquéia, bronquios e pulmão para mulheres

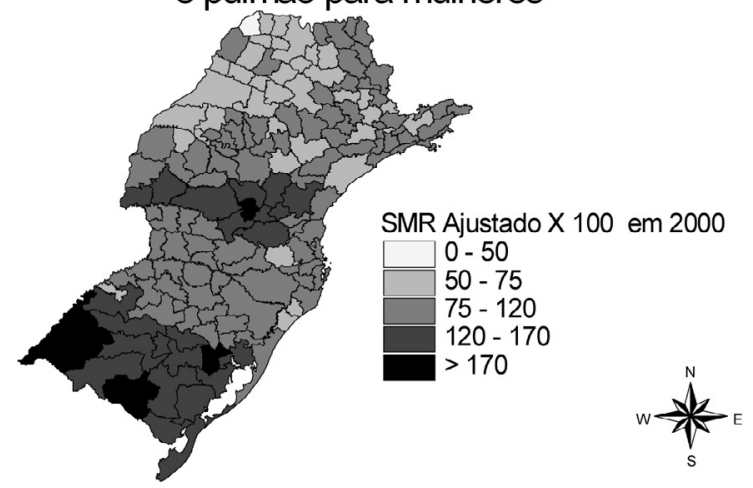

Figura 4 - Razões de Mortalidade Padronizada (SMR) ajustadas pelo modelo, mulheres, câncer de pulmão, sudeste e sul do Brasil, 1996 a 2000.

Figure 4 - Adjusted Standardized Mortality Ratio (SMR) for women, lung cancer, São Paulo and Southern Brazil, 1996 to 2000. 
Probabilidade do SMR ser maior que 1.5 para homens

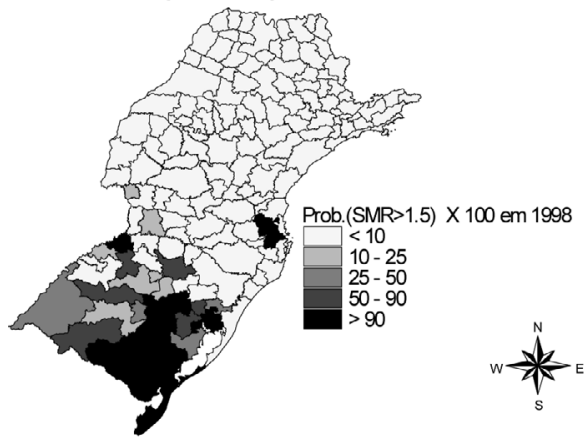

Probabilidade do SMR ser maior que 1.5 para mulheres

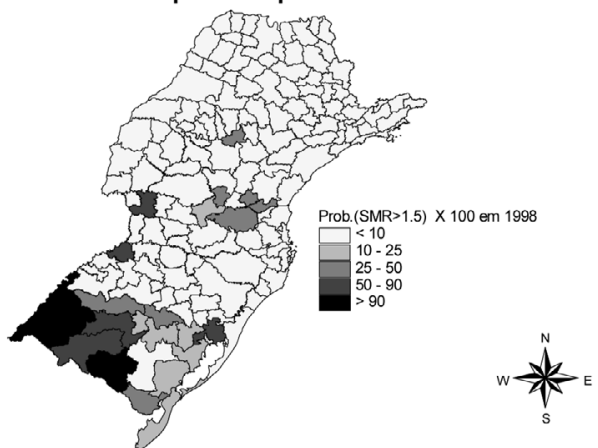

Figura 5 - Probabilidades a posteriori do SMR ajustado ser maior do que 1,5, homens e mulheres, câncer de pulmão, São Paulo e sul do Brasil, 1998.

Figure 5 - Posterior probabilities of adjusted SMR larger than 1,5, men and women, lung cancer deaths, São Paulo and South Brazil, 1996 to 2000.

\section{Percentual de crescimento da taxa em 5 anos para homens}

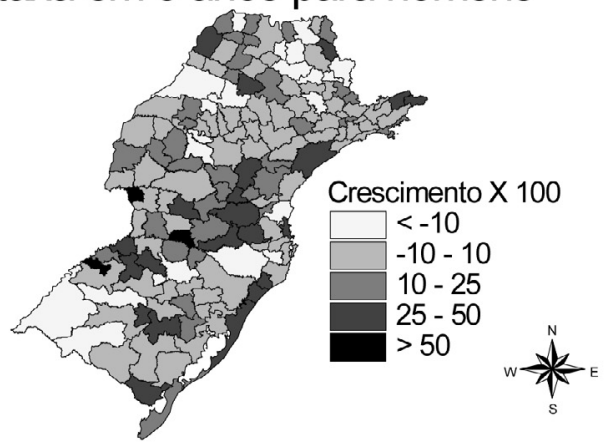

Crescimento anual das taxas para homens

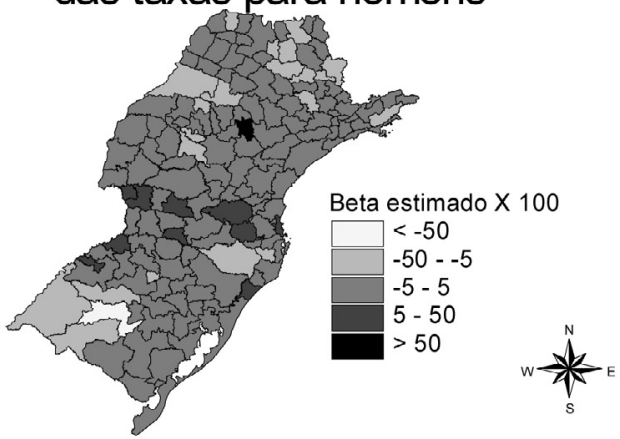

Percentual de crescimento da taxa em 5 anos para mulheres

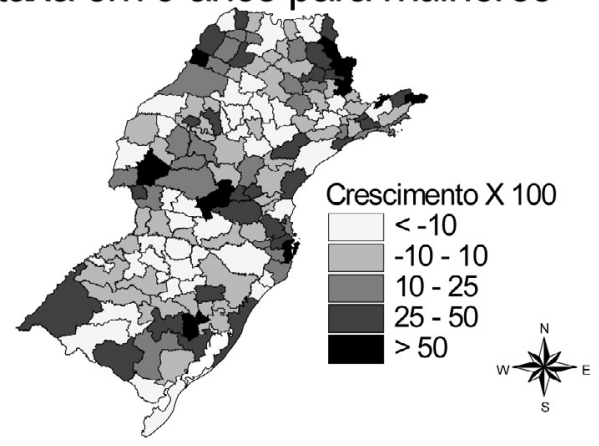

Crescimento anual das taxas para mulheres

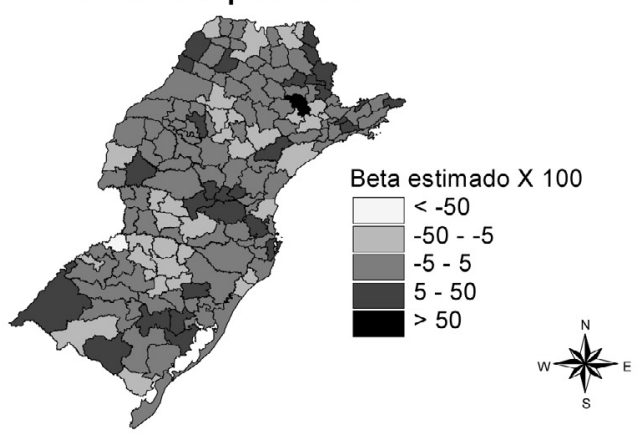

Figura 6 - Ritmo de crescimento do SMR e valores estimados para o coeficiente beta, homens e mulheres, câncer de pulmão, São Paulo e sul do Brasil, 1996 a 2000.

Figure 6-SMR increase pattern and estimated beta coefficients values, men and women, lung cancer deaths, São Paulo and South Brazil, 1996 to 2000. 
Capanema - PR e Cerro Largo - RS para homens e São João da Boa Vista, Batatais, Mogi-Mirim, Campos do Jordão, Bananal e Dracena (SP); Cascavel e União da Vitória (PR), Florianópolis - SC e São Jerônimo - RS para mulheres. Na Figura 6 também são apresentados os coeficientes beta, que representam o ritmo de crescimento ou decrescimento anual, e pode-se ver que não houve um gradiente espacial nítido para este ritmo. Para homens, as taxas se concentram na faixa de -5 a 5 , o que indica que a maior parte da região não possui crescimento ou decrescimento importante das taxas. As exceções importantes são as microrregiões de Santa Maria (RS), que apresentou um decrescimento de $94 \%$ nas taxas, e de Wenceslau Braz (PR), que apresentou um crescimento de $95 \%$ nas taxas. Para as mulheres, também foram encontradas duas microrregiões discrepantes, sendo elas Três Passos (RS), que teve uma queda da taxa de $70 \%$, e Piracicaba (SP), que teve crescimento da taxa de $93 \%$. É possível perceber ainda que o mapa para homens é mais homogêneo que para mulheres.

\section{Discussão}

O padrão espacial encontrado mostrou que no sul do Brasil, para homens, existiu um padrão em "U" nas razões de mortalidade por câncer de pulmão. Assim, as microregiões localizadas ao norte e ao sul da região estudada possuíam as maiores SMR e aquelas localizadas ao centro possuem SMR menores. Para mulheres, as maiores razões se localizaram no sul do Rio Grande do Sul e no Paraná. A principal hipótese para estes resultados seria a existência de maiores incidências de tabagismo nestas regiões, mas a inexistência desta informação no nível micro-regional impediu que esta variável fosse incluída na análise. Além disso, como o câncer de pulmão apresenta um longo período de latência, o ideal seria incluir não a informação sobre o tabagismo presente, mas a do tabagismo passado. Chama a atenção a concentração das maiores SMR nas micro-regiões próximas à fronteira com
Uruguai e Argentina, países que também apresentam alta taxa de mortalidade por câncer de pulmão ${ }^{6,} \mathrm{o}$ que poderia ser explicado por padrões similares de tabagismo para essas regiões.

Não houve um gradiente temporal único para o risco relativo de óbito por câncer de pulmão, sendo que algumas áreas apresentaram diminuição e outras apresentaram crescimento das razões. Este resultado pode estar relacionado a mudanças no padrão de tabagismo ao longo dos anos que antecederam o período estudado. Uma outra hipótese seriam questões ligadas ao sistema de informação ou ao sistema de diagnóstico e tratamento dos doentes. É importante ressaltar que a maioria das áreas não apresentou modificações temporais importantes, o que poderia ser explicado pelo período estudado, de apenas cinco anos.

Não existiu coincidência das micro-regiões que tiveram grande probabilidade do SMR ser maior do que 1,5 com aquelas que tiveram percentual de crescimento maior do que 50\%. É interessante notar que mais áreas para homens tiveram probabilidade do SMR ser maior do que 1,5 e mais áreas para mulheres apresentaram crescimento maior do que $50 \%$. Esta diferença pode ser atribuída ao fato de que o hábito de fumar está aumentando entre as mulheres em períodos mais recentes, diferentemente do que ocorreu para homens, ou seja, a epidemia de câncer de pulmão em mulheres se encontra em uma fase mais precoce do que a de homens ${ }^{5}$. Chamou a atenção o fato de que as maiores áreas urbanas destes Estados, com exceção de Porto Alegre e Florianópolis, não estavam incluídas nestas áreas de taxas altas e crescimento acelerado. Isto pode estar indicando uma maior estabilidade das taxas nas grandes áreas urbanas, seja por possuírem maior população e sistemas de informação já consolidados ou pelo fato de tanto a epidemia de tabagismo quanto o tratamento para o câncer de pulmão já estarem estabelecidos nestas áreas.

Em artigo que buscou agrupar os Estados brasileiros de acordo com a mortalidade por neoplasias ${ }^{17}$, os principais elementos 
discriminantes entre os Estados foram justamente neoplasias malignas da traquéia, brônquio e pulmão, embora também não se possa afirmar se estas diferenças tenham sido devidas a diferentes incidências de tabagismo ou a diferentes níveis de efetividade dos sistemas de saúde e/ou de cobertura dos sistemas de informação em saúde.

Os resultados apresentados no presente artigo podem ser utilizados pelos planejadores de saúde para elaborar políticas públicas voltadas para a redução do tabagismo e para a melhoria da abordagem do câncer de pulmão centradas nas micro-áreas que tiveram maior mortalidade, tanto do ponto de vista do maior crescimento temporal quanto das áreas que apresentaram as maiores razões de mortalidade.

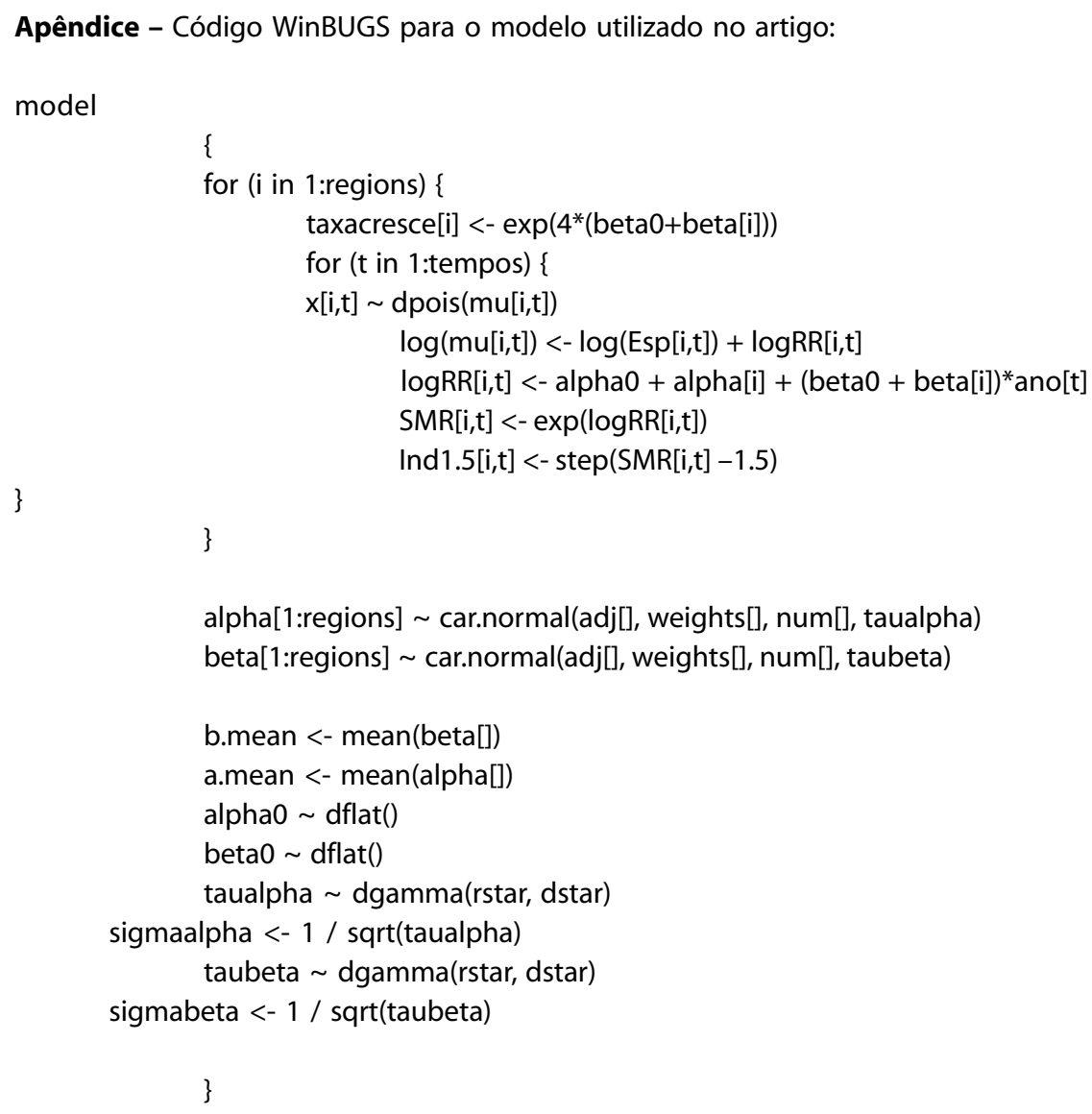

\section{Referências}

1. Ministério da Saúde. Anuário Estatístico da Saúde do Brasil - 2001. Brasília: Ministério da Saúde. http:// portal.saude.gov.br/saude/aplicacoes/anuario2001/ index.cfm; 2001.

2. Instituto Nacional do Câncer (INCA). Estimativa da Incidência e Mortalidade por Câncer no Brasil. Acessado em 08/10/2002. Disponível em http:// www.inca.gov.br/cancer/ epidemiologia/ estimativa2002/; 2002.
3. Blot WJ. The Epidemiology of Cancer. In: Bennet JC, Plum F, eds. Cecil Textbook of Medicine. Philadelphia: W. B. Saunders Company; 1996. p. 1020-4.

4. Instituto Nacional do Câncer (INCA). Prevenção e Detecção: Fatores de Risco, Tabagismo, Alcoolismo, Hábitos Alimentares. Disponível em http:// www.inca.gov.br/ cancer/prevenção/; 2002a.

5. Algranti E, Menezes AMB, Achutti AC. Lung Cancer in Brazil. Seminars in Oncology 2001; 28(2): 143-52. 
6. Marigo C. Câncer na América Latina - Perspectivas Sombrias. Rev Bras Cancer 1995; 41(2): 63-74.

7. Wünsch-Filho V \& Moncau JE. Mortalidade por Câncer no Brasil 1980-1995: Padrões Regionais e Tendências Temporais. Rev Assoc Méd Bras 2002; 48(3): 250-7.

8. Toledano MB, Jarup L, Best N, Wakefield J, Elliott P. Spatial variation and temporal trends of testicular cancer in Great Britain. Br J Cancer 2001; 84(11): 1482-7.

9. Jarup L, Best, N, Toledano MB, Wakefield J, Elliot P. Geographical epidemiology of prostate cancer in Great Britain. Int J Cancer 2002; 97: 695-9.

10. Short M, Carlin BP, Bushhouse S. Using Hierarchical Spatial Models for Cancer Control in Minnesota (Technical Report), 2002.

11. Assunção RM, Barreto, SM, Guerra, HL, Sakurai E, 1998. Mapas de taxas epidemiológicas: uma abordagem Bayesiana. Cad Saúde Pública 1998; 14(4): 713-23.

12. Carvalho MS, Câmara G, Cruz OG, Correa, V. Análise de Dados de Área. In: Druck, S., Carvalho MS, Câmara G, Monteiro A.M.V. Análise Espacial de Dados Geográficos. Disponível em: http://www.dpi.inpe.br/ gilberto/livro/analise/capitulo5-areas.pdf.
13. Bernardinelli L, Clayton D, Pascutto C, Montomoli C, Ghislandi M, Songini M. Bayesian analysis of spacetime variation in disease risk. Stat Med 1995; 14: 243343.

14. Assunção RA, Reis IA, Oliveira CL. Diffusion and prediction of Leishmaniasis in a large metropolitan area in Brazil with a Bayesian space-time model. Stat Med 2001; 20: 2319-35.

15. WinBUGS with DoodleBUGS. Version 1.4. Imperial College \& MRC, UK. Disponível em http://www.mrcbsu.cam.ac.uk/bugs/winbugs/contents.shtml

16. Clayton DE, Kaldor J. Empirical Bayes estimates of agestandardized relative risks for use in disease mapping. Biometrics 1987; 43: 671-81.

17. Pinto FG, Curi PR. Mortalidade por neoplasias no Brasil (1980/1983/1985): agrupamento dos Estados, comportamentos e tendências. Rev Saúde Pública 1991, 25(4): 276-81.

recebido em: $21 / 10 / 03$

versão reformulada apresentada em: 30/03/04

aprovado em: 05/04/04 